\title{
A facetless laser suitable for monolithic integration
}

\author{
D. Byrne' ${ }^{1}$, Q. Lu ${ }^{1}$, W. H .Guo ${ }^{1}$, J. F. Donegan ${ }^{1}$, B. Corbett ${ }^{2}$, B. Roycroft ${ }^{2}$, P. Lambkin ${ }^{2}$, J-P Engelstaedter ${ }^{2}$, \\ and F. Peters ${ }^{2}$ \\ 1. School of Physics and Centre for Telecommunication Value-Chain Driven Research (CTVR), Trinity College, Dublin 2, Ireland. \\ 2. Tyndall National Institute and Centre for Telecommunication Value-Chain Driven Research (CTVR), Cork, Ireland. \\ byrnede@tcd.ie, luqi@tcd.ie.
}

\begin{abstract}
A novel facetless laser structure is proposed and demonstrated. The facetless laser is realised by etching deep slots into the mirror sections of a multi section semiconductor laser which provide the feedback for laser operation.

OCIS codes: 140.5960 (Semiconductor lasers), 140.2020 (Diode lasers)
\end{abstract}

Introduction: Fabry-Pérot semiconductor lasers operate by injecting current into an active medium in a waveguide which produces and amplifies light. Lasing operation requires optical feedback which is conventionally obtained in a Fabry-Perot cavity by cleaving the ends of the waveguide forming two semi-reflecting facets. However due to the need for cleaving, it is difficult to integrate multiple components onto a single chip. Here we propose and demonstrate single mode lasing by etching slots into the waveguide, which provide the necessary reflectivity for laser operation. With such a design the need for cleaved facets is removed and such a laser can then be used as part of an integrated source.

The simulation and experimental results presented here are of a three section ridge waveguide laser with nine slots etched for each mirror section and a central gain section. Each mirror section has a different slot period giving a slightly different free spectral range (FSR). Independent injection of current into each section allows control of the optical gain and the refractive index of the sections. Due to the slots a periodic reflection spectrum is obtained from each section and changes in the refractive index in these sections causes an overlap of different reflection peaks providing a Vernier tuning mechanism of the output wavelength of the laser as in [1].

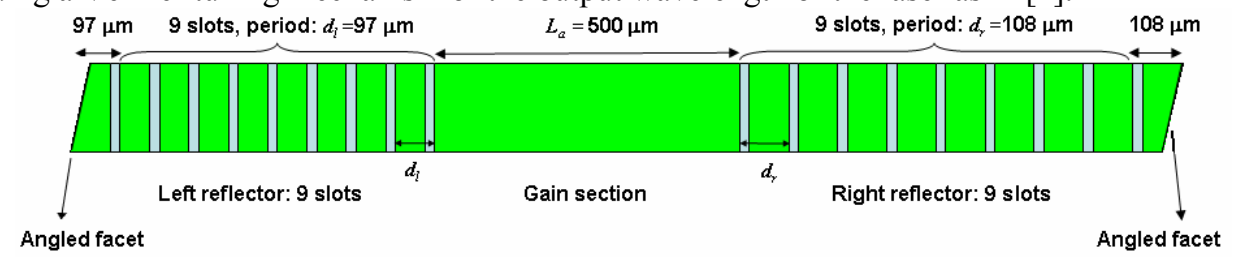

Fig. 1 Three section facetless laser structure.

Results: In order to know the amount of reflection from an individual slot we model the slot behaviour using a 2Dscattering matrix model (SMM) [2]. The dependence of the reflection from a single slot on slot depth is depicted in fig.2.

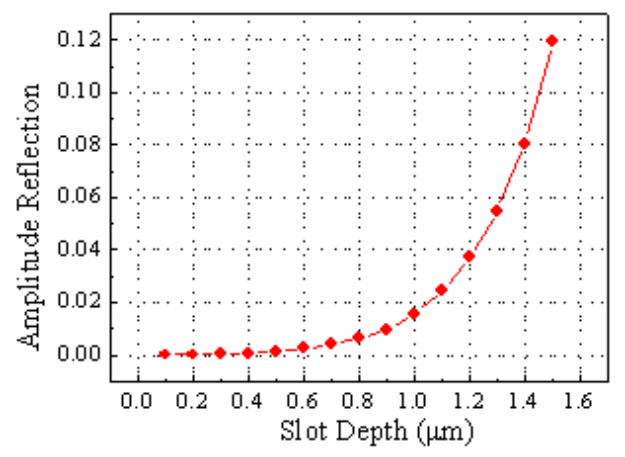

Fig. 2. Dependence of reflection on slot depth.

In order for lasing to occur the spectrally dependent reflection peaks from each mirror section must overlap resulting in strong feedback at this wavelength as the round-trip loss is inversely proportional to the product of both mirror reflectivities.

Fig. 3 shows modelling results of both reflection spectra one with an FSR of $3.2 \mathrm{~nm}$ and one with an FSR of $3.5 \mathrm{~nm}$ showing an overlap at $1510 \mathrm{~nm}$. Therefore at this wavelength the round-trip loss is overcome and lasing is possible. 


\section{OWQ6.pdf}

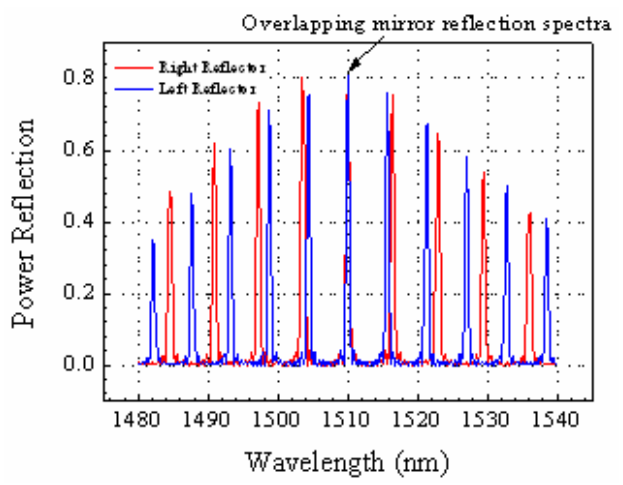

The slots in our lasers are etched to the top of the p-waveguide region, but do not penetrate into the quantum well active region. The total length of the cavity $\mathrm{L}$ is $\sim 2345 \mu \mathrm{m}$, however from Fig. 3 the longitudinal mode spacing $(\Delta \lambda)$ is determined to be $0.25 \mathrm{~nm}$ and from equation 1 we get an effective optical cavity length of $1250 \mu \mathrm{m}$, where $\lambda$ is the wavelength of the longitudinal mode. This confirms that the facets do not contribute to the laser operation. A FabryPérot laser of length $2345 \mathrm{~nm}$ with cleaved facets would give a mode spacing of $0.15 \mathrm{~nm}$.

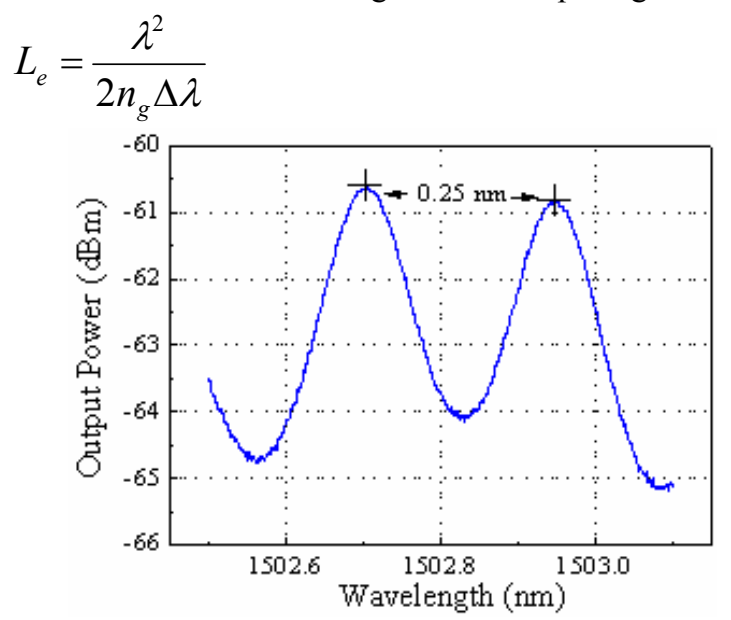

Fig. 3. Longitudinal mode spacing of the facetless laser.

This laser is designed to access a large number of wavelengths by changing the refractive index by injecting carriers into the independent mirror sections thus changing which of the peaks in the reflection spectra overlap. By setting the gain section injection current and one of the mirror injection currents and changing the other mirror injection current we can achieve discontinuous tuning over a few modes. By changing both mirror sections simultaneously we can access many more modes. The laser is fabricated as designed with nine slots in each mirror section and cleaved facets, these facets are subsequently angled using a focused ion beam (FIB) to remove the reflections from the facets and guaranteeing a facetless configuration.

Fig 4 shows the SMSR versus wavelength as the mirror injection currents are tuned brining different mirror reflection peaks into alignment for the laser prior to the angling of the facets, and Fig. 5 shows the SMSR versus wavelength after the facets have been angled. We can clearly see that there is no deterioration in the laser output after removing the facet reflection confirming that the laser is operating in a facet free form. We measure 11 accessible modes with SMSR of over $30 \mathrm{dBm}$ with longitudinal mode spacing of $\sim 3 \mathrm{~nm}(400 \mathrm{GHz})$. Fig 6 shows some of these lasing modes. Very good single mode operation is observed. 


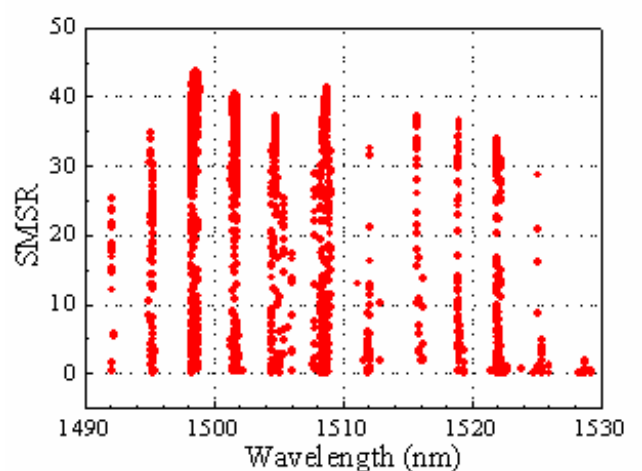

Fig. 4. SMSR versus Wavelength for three section cleaved facet laser.

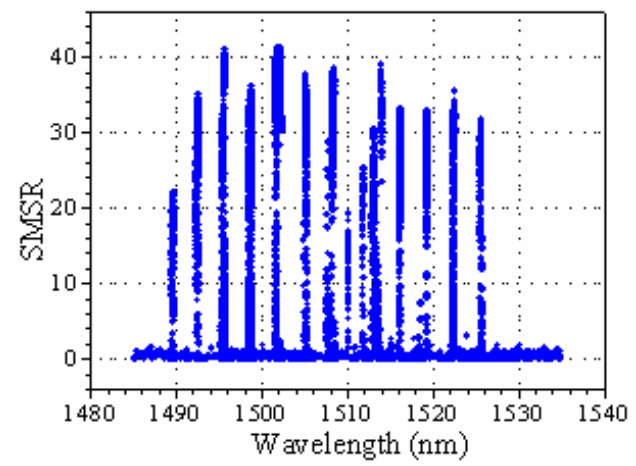

Fig. 5. SMSR versus Wavelength for three section angled facet (facetless) laser.

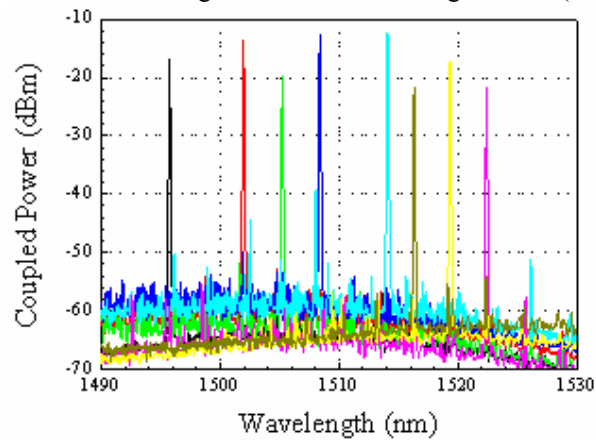

Fig. 6. Lasing spectra for facetless laser.

Conclusion: We have demonstrated tuneable single mode lasing of a facetless laser realised by etching slots into a ridge waveguide and using the Vernier tuning mechanism. We have verified that the laser is indeed operating independent of the cleaved facets by angling the facets to remove any reflection from them. We have achieved single mode lasing with SMSR of over $40 \mathrm{~dB}$ and access 11 modes.

Acknowledgement: This work was supported by Science Foundation Ireland under the CTVR programme References:

[1] Phelan, R. Guo, W. H. Lu, Q. Y. Byrne, D. Roycroft, B. Lambkin, P. Corbett, B. Donegan, J. F. "A Novel Two-Section Tunable Slotted Fabry-Perot Laser Exhibiting ns Wavelength Switching." Transparent Optical Networks, 2007. ICTON '07. 9th International Conference on, Vol 2, 1-5 July 2007, pp 124-127.

[2] Q. Y. Lu, W. H. Guo, R. Phelan, D. Byrne, J. F. Donegan, P. Lambkin, and B. Corbett, "Analysis of Slot Characteristics in Slotted SingleMode Semiconductor Lasers Using the 2-D Scattering Matrix Method" IEEE Photonics Technology Letters, vol. 18, no. 24, December 15, 2006. 